\title{
XXXII. Admittance and impedance loci
}

\section{Frederick Bedell}

To cite this article: Frederick Bedell (1896) XXXII. Admittance and impedance loci, Philosophical Magazine Series 5, 42:257, 300-308, DOI: 10.1080/14786449608620923

To link to this article: http://dx.doi.org/10.1080/14786449608620923

$$
\text { 曲 Published online: } 08 \text { May } 2009 .
$$

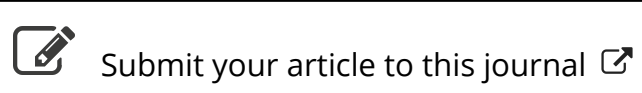

\footnotetext{
Џ Article views: 4
}

Q View related articles ¿ 
where $\left(\frac{\varpi_{0}}{\sigma_{0}}\right)_{\mathrm{H}}$ is the value for hydrogen at $0^{\circ}$, and this is A $\frac{A}{.0000896}$ where $A=1$ atmo.

Also $\mathrm{NM}=1000 p$.

Substituting these values we obtain

$$
\begin{aligned}
\mathrm{P} & =\frac{n}{\mathrm{~N}} \cdot \frac{2}{\mathrm{M}} \cdot \frac{\mathrm{A} \rho(1+a t)}{0 \cdot 0000896} \\
& =\frac{2 n \mathrm{~A}}{0.0896}(1+\alpha t) \\
& =22 \cdot 3 n \mathrm{~A}(1+\alpha t) .
\end{aligned}
$$

If $a$ has any other value than 1 we must put

$$
\mathrm{P}=22 \cdot 3 \operatorname{an} \mathrm{A}(1+\alpha t) \text {, }
$$

whence we see that if each salt molecule combines with two or with three solvent molecules the osmotic pressure is double or treble the normal value.

The supposition here made is no doubt crude in its simplicity, but my attempts to introduce other considerations, such as change in density in the solution, have led to such complicated results that much more extravagant suppositions had to be made to reconcile these results with experiments. I therefore leave the hypothesis in this crude form, in which it will at least serve to show that it is not necessary to ascribe osmotic pressure to dissociation but rather to association or some kind of combination of salt and solvent.

\section{Admittance and Impedance Loci. By Frederick Bedell*.}

1 THE quantities chiefly considered in the discussion of alternating currents are electromotive forces and currents, the values of these being determined for different conditions. Electromotive forces and currents are commonly represented by vector diagrams ; and the change in these diagrams, as some one quantity is varied, is shown by the loci of the vectors which are altered thereby. What may be termed electromotive force and current loci are thus determined. The numerical values for which these are consiructed necessarily depend upon some condition involving an assumed

* Communicated by the Physical Society: read June 26, 1896. 
value either of the current or of the electromotive force : thus we may assume a certain current to be constant (as the primary current of a transformer), and construct an electromotiveforce diagram with loci showing the changes in the various electromotive forces as some part of the circuit is changed; or we may assume the impressed electromotive force constant, and ascertain current loci for the same variation. Let us limit ourselves to the transformer. In the first case above it will be found that the constant assumed primary current $I_{1}$ is a factor in the value of every line representing the components of the primary electromotive force $\mathrm{E}_{1}$. By factoring out $I_{1}$ we have an impedance diagram similar to the electromotive-force diagram, and without any assumption as to the value of the current or electromotive force. Similarly each line in a current diagram, constructed for a constant inpressed electromotive force $E_{1}$, represents a current which is a multiple of an admittance (the reciprocal of an impedance) and the factor $\mathrm{E}_{1}$. By factoring out $\mathrm{E}_{\mathrm{i}}$ an admittance diagram is consequently obtained, similar to the current diagram but with no assumption as to the current or electromotive force. Admittance and impedance diagrams accordingly correspond to current and electromotive-force diagrams respectively, differing from them only by a factor.

Impedance and admittance loci, or electromotive force and current loci, for the primary of a transformer will in general be ares of circles for changes in any one of the constants of the primary or the secondary circuit.

Some interesting relations arise from the reciprocal naturo of admitiance and impedance. Let us note the following relations between reciprocal vectors :-

If any vector has an arc of a circle for its locus, a vector proportional to its reciprocal will have an arc of a circle for a locus. In fig. 1 let $\rho_{1}$ be any vector from the origin 0 , having its locus as shown upon the arc of a circle. The vector $\rho_{2}$, drawn in the direction of $\rho_{1}$ and proportional to its reciprocal, will have its locus upon an arc of a circle, which mar be shown as follows. Let $\rho_{1}$ and $\rho_{1}^{\prime}$ represent the vector in any two positions, $\mathrm{OA}$ and $\mathrm{OA}^{\prime}$. 'The intercepts $\mathrm{O} a$ and $\mathrm{Oa}^{\prime}$ will represent the reciprocal vectors $\rho_{2}$ and $\rho_{2}{ }^{\prime}$; for in the siruilar triangles $\mathrm{OA}^{\prime} a$ and $\mathrm{OA} a^{\prime}$,

Hence

$$
\rho_{1}: \rho_{1}^{\prime}:: \rho_{2}^{\prime}: \rho_{2} \text { 。 }
$$

$$
\rho_{1}^{\prime} \rho_{2}^{\prime}=\rho_{1} \rho_{2}=\text { a constant. }
$$

The value of this constant product of $\rho_{1}$ and $\rho_{2}$ is $\overline{O G}^{2}$. 
Fig. 1.-Reciprocal Vectors, $\rho_{1}$ and $\rho_{2}$.

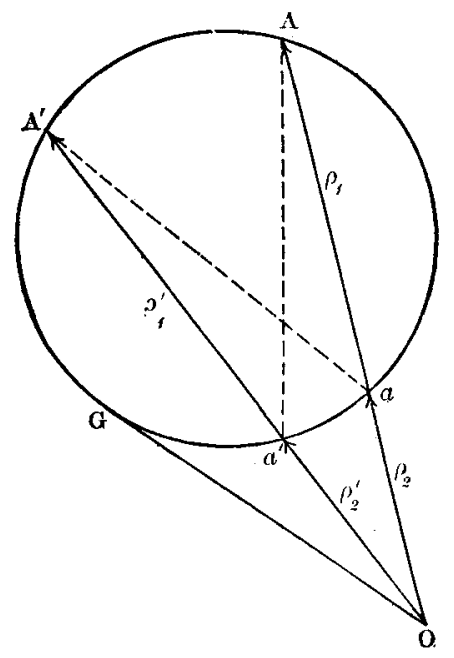

Fig. 2.-Reciprocal Vectors.

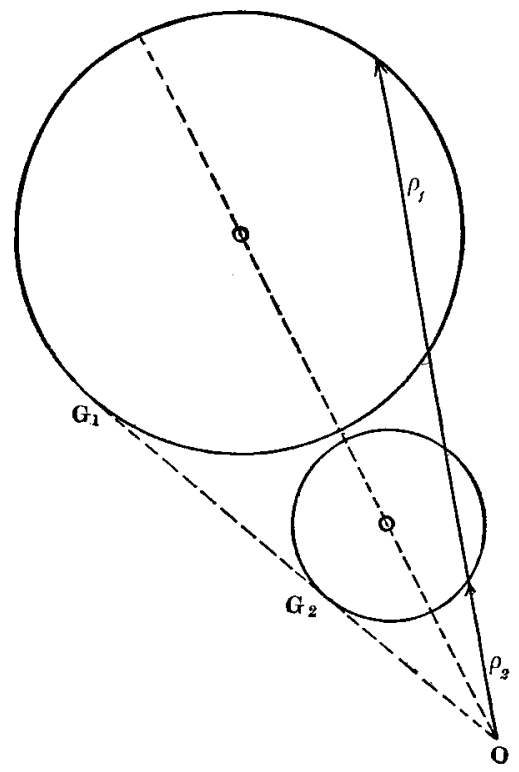

By a suitable selection of scale, the loci of $\rho_{1}$ and its reciprocal $\rho_{2}$ may be represented as arcs of the same circle, as in 
fig. I; or they may be represented by different circles, as in fig. 2. In the latter case,

$$
\rho_{1} \rho_{2}=O \mathrm{OG}_{1} \cdot O \mathrm{G}_{2}=\mathrm{a} \text { constant. }
$$

As the origin $O$ approaches the circle which represents the locus of $\rho_{1}$, the centre of the reciprocal circle becomes more distant and its radius becomes greater. When the origin $O$ is a point in the circumference of the first circle, the centre of the reciprocal circle is at an infinite distance; that is, the reciprocal locus is a straight line.

Let us apply these principles to the transformer diagrams. The locus of the primary impedance, as some particular quantity is varied, is a portion of a circle. For example, this may be shown to be the case when the secondary resistance is varied. Since the admittance of the primary is the reciprocal of its imped:nce, the admittance may be represented by the vector $\rho_{2}$ in the above construction, if the impedance is represented by $\rho_{1}$. These loci may be drawn to scale for actual values. In a constant current transformer the primary elnctromotive force varies directly as the primary impedance. In a constant potential transformer the primary current varies directly as the primary admittance. But the admittance is the reciprocal of the impedance; hence if we have an arc of a circle for the locus of the primary electromotive force when the primary current is maintained constant, we may employ the above method to obtain the arc of a circle which will be the locus for the primary current when the transformer is supplied with a constant electromotive force. The converse operation may likewise be performed.

In fig. 3 let the circle $\mathrm{C}_{1}$ represent the locus of the primary electromotive force $\mathbb{F}_{1}$ during some particular change of condition, the primary current meanwhile being maintained constant and in the direction $\mathrm{OA}$. The difference in phase between the current and electromotive force is the angle $\phi_{1}$. The locus of the primary current under the same change of conditions, if the primary electromotive force is maintained constant, is the dotted circle $\mathrm{C}_{2}$, which is reciprocal to $\mathrm{C}_{1}$. If the constant electromotive force is drawn in the direction $\mathrm{OA}$, the locus of the primary current is the circle $\mathrm{C}_{2}{ }^{\prime}$, drawn so that the angles $\mathrm{AOC}_{1}$ and $\mathrm{AOC}_{2}{ }^{\prime}$ are equal.

An application of the method of reciprocal vectors is shown in fig. 4. Positive rotation is counter-clockwise. The semicircle JKN represents the locus of the primary electromotive. force of a transformer, when the primary current is constant 
Fig. 3.

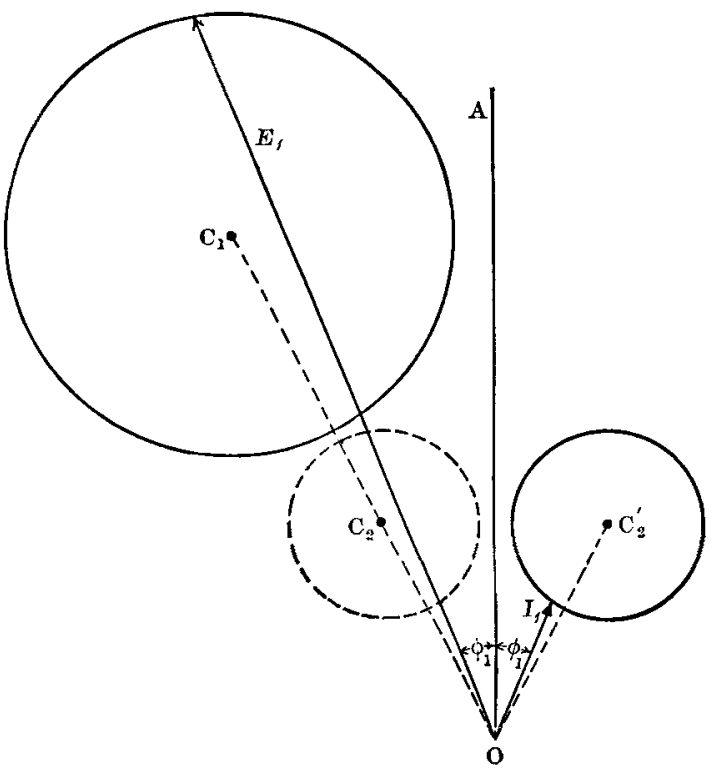

Fig. 4,-Nethod of obtaining Primary Current Locus by the Principle of Reciprocal Vectors.

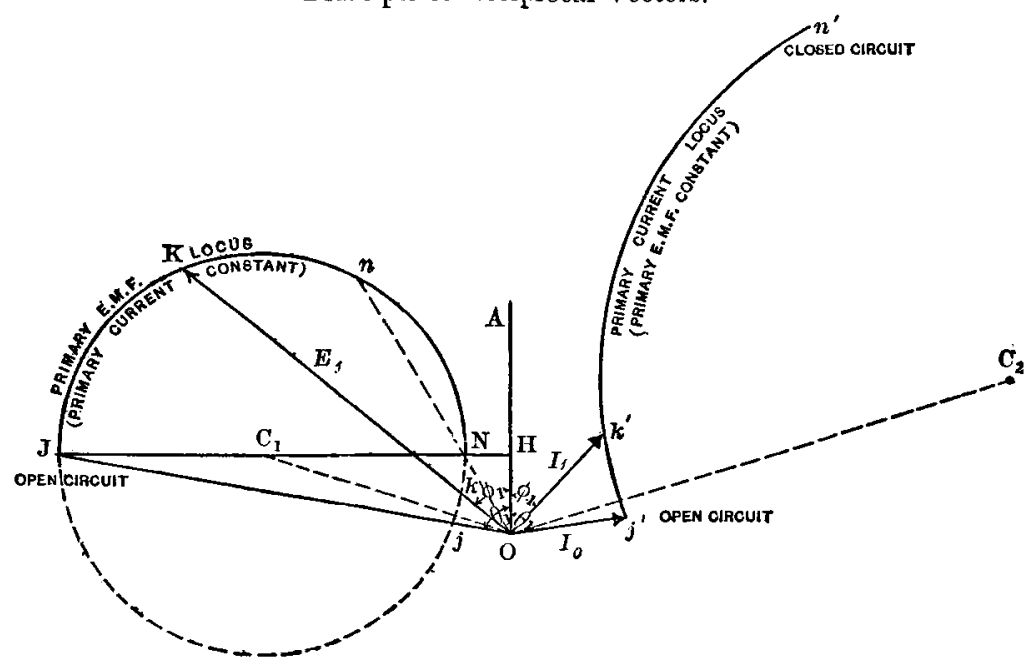

and is in the direction of $\mathrm{OA}$, and the secondary resistance is varied. 
The electromotive force has the position $\mathrm{OJ}$ on open circuit and $\mathrm{ON}$ on short circuit. $\mathrm{OH}$ is the power electromotive force on open circuit, and includes the effects of primary resistance and the losses due to hysteresis and eddy currents; HJ is the electromotive force to overcome the primary selfinduction. These are in the direction of the primary current, and at right angles to it, respectively. A line from $J$ to $K$, at right angles to the secondary current, would show the reaction of the secondary upon the primary. It is to be noted that the line $\mathrm{NH}$ represents the effects due to magnetic leakage. It is desired to find the locus for the primary current when the primary electromotive force has a constant value, and is drawn in the direction $O A$. The :mgle of lag $\theta_{1}$, between the primary electromotive force and current on open circuit, is $\mathrm{JOH}$. Accordingly, with a constant electromotive force in the direction $O A$, the open circuit current $I_{0}$ is laid off lagging behind the electromotive force at an angle of $\mathrm{AO} j^{\prime}=\theta_{1}=\mathrm{JOH}$. The open circuit current $\mathrm{I}_{0}$ may be laid off to any convenient scale. To construct the locus for the primary current proceed as follows:-Lay off the line $\mathrm{OC}_{2}$ so that the augles $\mathrm{AOC}_{1}$ and $\mathrm{AOC}_{2}$ are equal. The point $\mathrm{C}_{2}$ is located so that $\mathrm{OC}_{2}: \mathrm{OC}_{1}:: \mathrm{Oj}^{\prime}: O j$. The primary current locus is then drawn as the are of a circle with $\mathrm{C}_{2}$ as a centre, passing through $j^{\prime}$.

The limits of the primary electromotive force locus are the points $\mathrm{J}$ and $\mathrm{N}$. The corresponding limits of the primary current locus are the points $j^{\prime}$ and $n^{\prime}$. It will be noted that these points correspond to the points $j$ and $n$ on the circle $\mathrm{C}_{1}$, which are reciprocal to the points $\mathbf{J}$ and $\mathrm{N}$.

In the ahsence of magnetic leakage the points $\mathrm{N}$ and $\mathrm{H}$ coincide. The point $n^{\prime}$ would then lie in the line OA. The deviation of the primary current locus from the line $O A$ is produced by magnetic leakage.

An experimental curve showing the primary current locus for a constant potential transformer, as aff ${ }^{\mu}$ cted by magnetic leakage, is shown in fig. 5 .

The reciprocal relation between admittance and imped:ınce vectors gives a simple method for determining the conditions for consonance and resonance in transformer circuits*.

Figure 6 is given as a particular instance in illustration of the statement given above that loci produced by the variation of any one constant are usually ares of circles. 'The primary loci are always arcs of circles. The diagram shows the changes due to a variation in the secondary self-induction.

* "Resonance in Transformer Circuits," by Bedell and Crehore. "The Plyssical Review, vol. ii. p. 442.

Phil. Mag. S. 5. Vol. 42. No. 257. Oct. 1896. 
Fig. 5.-Primary Current Locus for Constant Potential 'Transformur: Determined Experimentally.

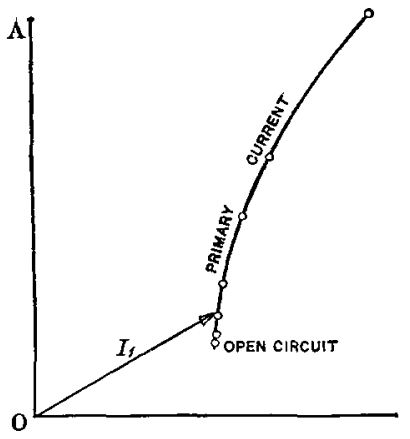

Fig. 6.-Effect of the Variation of the External Secondary Self-Induction in a Constant Current Transformer.

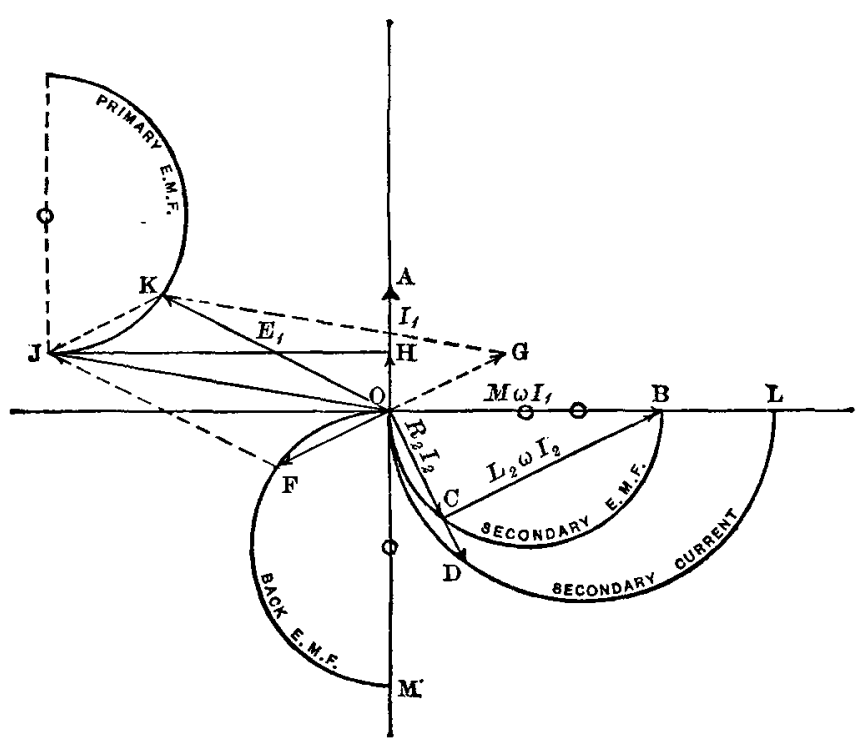

Fig. 7 shows the effect of magnetic leakage. The curves shown are loci for primary electromotive force, when the primary current is $\mathrm{I}_{1}$. The primary electromotive force is composed of the components $\mathrm{OH}$, to overcome ohmic resistance and supply open-circuit losses, HJ to overcome selfinduction, and (with absence of magnetic leakage) $\mathrm{JK}_{0}$ to overcome the back electromotive force of mutual induction. 
The semicircle $J_{0} \mathrm{H}$ is the locus for the primary electromotive force in the absence of magnetic leakage. The semicircle $J K^{\prime} N$ is the locus for the primary electromotive force

Fig. 7.--Effect of Magnetic Lealage.

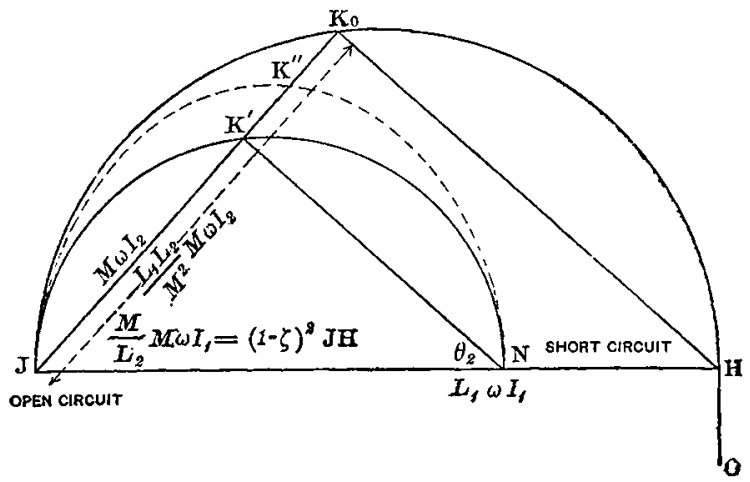

when the coefficient of magnetic leakage $\zeta$ is constant from open circuit to short circuit. In this case we have the relations

$$
\begin{aligned}
\frac{\mathrm{JK}^{\prime}}{\mathrm{JK}_{0}} & =\frac{\mathrm{JN}}{\overline{\mathrm{JH}}}=\frac{\mathrm{M}^{2}}{\mathrm{~L}_{1} \mathrm{~L}_{2}}=(1-\zeta)^{2} ; \\
1-\zeta & =\sqrt{\frac{\overline{\mathrm{JK}^{\prime}}}{\mathrm{JK}_{0}}=\sqrt{\frac{\mathrm{JN}}{J \mathrm{H}}} ;} \\
\zeta & =1-\sqrt{\frac{\mathrm{JK}^{\prime}}{\overline{J K}_{0}}}=1-\sqrt{\overline{\mathrm{JN}}} .
\end{aligned}
$$

In an actual transformer the magnetic leakage is not constant, but varies with the load. The locus represented by the dotted curve $J K^{\prime \prime} \mathrm{N}$ is for such a case in which the magnetic leakage is zero on open circuit and increases to the maximum at short circuit. Where the magnetic leakage is variable, it is determined for any point as $\mathrm{K}^{\prime \prime}$ by the ratio of $\mathrm{JK}^{\prime \prime}$ to $\mathrm{JK}_{0}$.

$$
\zeta=1-\sqrt{\frac{J^{\prime \prime}}{J \bar{K}_{0}}} \text {. }
$$

Thus let us suppose that the back electromotive force JK". actually induced in the primary by the secondary current $\mathrm{I}_{2}$, is eighty-one volts; and that $J K_{0}$, which would be the back electromotive force in the absence of magnetic leakage, is 100 volts. We then have the equation

$$
1-\zeta=\sqrt{\frac{81}{100}}=\frac{9}{10}=0.9 \text {. }
$$


which indicates that the mutual induction is nine-tenths of the value it would have in the case of no magnetic leakage. The coefficient of magnetic leakage is accordingly found to be 10 per cent. ; thus :-

$$
\zeta=1-0 \cdot 9=0 \cdot 10 \text {. }
$$

Fig. 7 is drawn to represent the values of the various electromotive forces in the primary circuit of a transformer, for a given value of the primary current and for different values of the secondary resistance. If the magnitude of each line is divided by the primary current $I_{1}$, fig. 7 represents the values for the primary impedance (without any assumption as to constant current or electromotive force) for different values of the secondary resistance. The effect of magnetic leakage upon the primary impedance of any transformer is thus shown for different values of the secondary resistance.

The above construction affords a simple method of studying the conditions for a decrease of the primary impedance of a transtormer when the secondary circuit is closed *

Curves corresponding to the dotted curve in fig. 7 have been experimentally determined + by the witer.

Cornell University, May 1896.

XXXIII. Electric Images in the Field of a Hittorf's (Crookes') Tube. By N. Oumof F and A. SayoÏLOFf

THE influence exercised by a Hittorf"s tube on electrified bodies shows that the electric field created in the interior of the tube extends also to the exterior. Evidently the objects brought into this field, whether to explore its electrical properties or, as in the experiments of Lenard and Röntgen, to produce or to receive shadows acquire a new electrical condition and by their presence modify the primitive field. It is impossible to estimate $a$ priori this modification and the part it plays in the production of the phenomena observed. In investigating these questions we finally adopted an experimental method intended to furnish us with the means of forming a general idea of the electrical properties of the field of a Hittorf's tube and the modifications which it undergoes.

* Discussed at length before the Physical Society by Mr. E. C. Rimington in his paper "On Air-core Transformers," Philosophical Magazine, vol. xxxvii. p. 394.

+ 'Proceedings of the International Electrical Congress,' Chicago. 1893 , p. 234.

$\ddagger$ Communicated by Lord Kelvin, F.R.S. 